Recibido:

12-XI-2018

Aceptado:

17-|-2019

Publicado en línea:

$13-11-2019$
Bruxismo en niños y adolescentes: Revisión de la literatura

\section{Bruxism in Children and Adolescents: A Review of the Literature}

Viviana Abigail Alvarez-Gastañaga'; Mercedes Carolina Baldeón-López;

Violeta Malpartida-Carrillo CD, Mg, Esp²

1. Estudiantes de Pregrado, Universidad Científica del Sur, Lima, Perú.

2. Especialista en Implantología Oral, Magister en Implantología Oral, Docente de pregrado y posgrado, Universidad Científica del Sur, Lima, Perú.

Autor para correspondencia: Dra. Violeta Malpartida-Carrillo - vmalpartida@cientifica.edu.pe

RESUMEN: El bruxismo se define como una actividad repetitiva de los músculos masticatorios caracterizada por apretar o rechinar los dientes y/o por golpear y balancear la mandíbula. Es de etiología multifactorial y se considera que existe un bruxismo fisiológico necesario para el desarrollo de la cara de los niños. Estos dos aspectos hacen complicado el diagnóstico que se basa fundamentalmente en un cuestionario a los padres y en el examen clínico del niño. Cuando el desgaste fisiológico no se diferencia a tiempo del desgaste parafuncional, trae consecuencias negativas en el niño que alteran su calidad de vida, necesitando un manejo multidisciplinario adecuado. Considerando que es necesario conocer características fundamentales del bruxismo infantil, el objetivo del presente artículo de revisión es realizar una actualización sobre sus manifestaciones circadianas, clasificaciones, prevalencia, etiología, factores de riesgo, diagnóstico y tratamiento que servirá al odontólogo para una temprana identificación y tratamiento.

PALABRAS CLAVE: Bruxismo; Bruxismo del sueño; Trituración; Niños; Adolescente. 
ABSTRACT: Bruxism is defined as a repetitive activity of the chewing muscles characterized by clenching or grinding of the teeth and/or by striking and swinging the jaw. It is of multifactorial etiology and it is considered that there is a physiological bruxism necessary for the development of the face of children. These two aspects complicate the diagnosis that is fundamentally based on a questionnaire to the parents and on the clinical examination of the child. When the physiological wear does not differentiate in time from the parafunctional wear, it has negative consequences in the child that alter their quality of life, requiring an adequate multidisciplinary management. Considering that it is necessary to know the fundamental characteristics of bruxism in children and adolescents, the objective of this review article is to update its circadian manifestations, classifications, prevalence, etiology, risk factors, diagnosis and treatment that will serve the dentist for the early identification and treatment.

KEYWORDS: Bruxism; Sleep bruxism; Grinding; Children; Adolescent.

\section{INTRODUCCIÓN}

Se define bruxismo a la actividad motora oral diurna o nocturna que consiste en el apriete 0 rechinamiento involuntario, rítmico o espasmódico no funcional de los dientes (1). La Academia Americana de Medicina del Sueño lo define como una actividad muscular mandibular caracterizada por apretar o rechinar los dientes y/o por refuerzos o empuje de la mandíbula durante el sueño (2).

El ruido producto del rechinamiento dentario es intenso, en repetidas ocasiones y por largo tiempo, convirtiéndose en una preocupación para los padres. Si es que no se identifica y trata a tiempo puede traer consecuencias negativas como desgaste de los dientes, enfermedad periodontal, hipertrofia de los músculos masticatorios, cefalea, sensación de limitación de la apertura bucal, dolor muscular y trastornos temporomandibulares (TTM) que finalmente disminuyen el rendimiento académico del niño y del adolescente $(3,4)$.

El bruxismo es un importante problema de salud público que tiene un impacto negativo en la calidad de vida de los niños y adolescentes que lo presentan y debe ser conocido por el odontólogo para diagnosticarlo a tiempo, identificar los factores de riesgo, educar a los padres y prevenir sus posibles consecuencias en la salud bucal (5-7).

Por tal motivo, el objetivo de la presente revisión es realizar una actualización sobre sus manifestaciones circadianas, clasificaciones, prevalencia, etiología, factores de riesgo, diagnóstico y tratamiento que servirán al odontólogo para una temprana identificación, orientación y manejo.

\section{MANIFESTACIONES CIRCADIANAS}

Presenta dos manifestaciones circadianas, una ocurre durante el sueño y se conoce como bruxismo del sueño (BS) o bruxismo dormido y la otra, ocurre durante la vigilia y se conoce como bruxismo despierto (BD) o bruxismo en alerta $(3,8,9)$. El BS se caracteriza por apretamiento y rechinamiento dentario, mientras que el BD se presenta casi siempre solo apretamiento (10). Incluso, en algunos niños y adolescentes puede presentarse el bruxismo mixto.

\section{CLASIFICACIÓN}

El bruxismo se puede clasificar como primario o idiopático, cuando no hay comorbilidades médicas asociadas o causas claras identificables; 
secundario o iatrogénico, cuando se relaciona a condiciones psicosociales o médicas como los trastornos respiratorios del sueño, problemas neurológicos, condiciones psiquiátricas y el consumo de drogas o medicamentos $(11,12)$. También se ha clasificado como bruxismo céntrico o de apretamiento, cuando existe presión sobre los dientes en posición de intercuspidación y está relacionado a la función anormal de los músculos masetero y temporal y bruxismo excéntrico, cuando existe presión fuera del área de oclusión habitual, relacionado a la función anormal los músculos masetero, temporal y pterigoideos (13). Fernández (7) clasifica al bruxismo como leve, cuando se manifiesta solo algunas noches y no existen evidencias de lesiones en los dientes; bruxismo moderado, cuando se manifiesta todas las noches y está asociado a un problema psicosocial leve y bruxismo grave, cuando se manifiesta todas las noches, existe evidencia de lesión en los dientes y está asociado a problemas psicosociales graves.

\section{PREVALENCIA}

Una revisión sistemática analizó 8 estudios realizados en Turquía, China, USA, Brasil, Taiwán y Finlandia reportando una prevalencia de 3.5\% a $40.6 \%$ en niños y adolescentes menores de 12 años, sin diferencias entre géneros y que va disminuyendo con la edad (14). Otra revisión sistemática reportó que la prevalencia era de 5\% a 40\% en niños menores de 10 años, analizando 4 estudios realizados en Brasil, China y USA (15).

La prevalencia del BS en niños y adolescentes de Latinoamérica es muy variable. En Brasil en el año 1998 Shinkai et al (16) reportaron una prevalencia de $43 \%$ entre niños de 2 a 3 años, de $35 \%$ entre 4 y 5 años y de $34 \%$ entre 10 y 11 años. Luego, se registraron prevalencias de $32 \%$ (17) y $35.7 \%$ (18). En el año 2012, Renner et al (19) encontraron una prevalencia de 39.1\% en edades de 7-9 años y de $35.7 \%$ en edades de 9-11 años. Recientemente, en el año 2016, se reportó una prevalencia de $32,4 \%$ en edades de 3 a 12 años (20). Así mismo, Sandoval et al (21) reportaron que en Chile la prevalencia de BS es del $32 \%$ y sin diferencias entre géneros, la mayor prevalencia se observó a los 6 años con el 38\% y la menor, a los 14 años con el $27 \%$.

El gran rango de discrepancia entre los porcentajes puede deberse a distintos conceptos 0 definiciones, diferentes instrumentos de medición utilizados para el diagnóstico, distintas características de las poblaciones y metodologías de investigación.

\section{ETIOLOGÍA}

Muchos investigadores creen que el bruxismo tiene origen multifactorial $(22,23)$. Según la literatura, la etiología del bruxismo puede dividirse en:

- Factores locales: Asociados a la presencia de TTM, traumatismos oclusales, maloclusiones, contactos prematuros, presencia de cálculo radicular, reabsorción radicular, pérdida prematura de dientes, quistes dentígeros, tensiones musculares y excesos de materiales restauradores $(24,25)$. También se reporta que en niños pequeños puede deberse a inmadurez del sistema masticatorio neuromuscular (26).

- Factores sistémicos: Asociados a deficiencias nutricionales y vitamínicas, parasitosis intestinales, alergias, parálisis cerebral, deficiencias mentales y disturbios endocrinológicos, otorrinolaringológicos y gastrointestinales $(16,27)$. También se ha asociado con enfermedades sistémicas y del sueño, incluyendo parasomnias y problemas del estado de ánimo $(5,18)$.

- Factores psicológicos: Asociados a trastornos neuroconductuales, problemas familiares, de personalidad, tensiones emocionales, crisis existenciales, estados de depresión, ansiedad, miedo y hostilidad (28). 
- Factores ocupacionales: Como por ejemplo pruebas escolares, práctica de deportes competitivos y campeonatos (26).

- Factores hereditarios: Algunas investigaciones reportan que los hijos de padres que tenían bruxismo en la infancia, aprietan o rechinan los dientes (29). También se menciona que los hijos de padres con bruxismo son más susceptibles al hábito (30).

- Factores fisiopatológicos: Relacionados a la química cerebral alterada. Según la literatura, el sistema nervioso central se ve estimulado por TTM, maloclusiones, hábitos orales, ansiedad 0 estrés y responde con alteraciones en la neurotransmisión de la dopamina, produciéndose una respuesta periférica de apretamiento y rechinamiento dental $(15,31)$.

\section{FACTORES DE RIESGO}

Una revisión sistemática reciente menciona que los factores de riesgo asociados al BS en niños son el hecho de que estén muy expuestos al humo de segunda mano (humo que proviene de la quema de un cigarrillo o cigarro), neuroticismo, trastornos del sueño y comportamientos parafuncionales (32). Así mismo, un metaanálisis reciente menciona como factores de riesgo la presencia de ronquidos, respiración bucal, sueño inquieto, babeo, posición del estómago y falta de sueño al acostarse (3). También se describe a la angustia, anomalías del comportamiento, trastornos del sueño (33), mioespasmos musculares, enuresis nocturna, cólicos, excesiva salivación nocturna, conversar dormido (34), succión digital, lengua protruida y respiración bucal (35) como posibles factores de riesgo.

\section{DIAGNÓSTICO}

En niños de 3 hasta 506 años se debe considerar el desgaste fisiológico ya que corresponde al período de abrasión y desgate preparatorio al recambio de los dientes deciduos. Debido a que en esta etapa el niño se encuentra en un período de gran plasticidad tisular de los tejidos óseo, dentario y neuroarticular, no menciona síntomas de dolor ni espasmos.

Se recomienda la integración de dos aspectos: cuestionario a los padres y examen clínico extra e intraoral del niño. En el cuestionario los padres responden la anamnesis para conocer antecedentes sobre desórdenes médicos, mentales o del sueño, consumo de fármacos, hábitos, quejas de dolor, relación familiar y social y para evaluar del perfil psicológico del niño y los datos específicos del rechinamiento dental (21). Se debe indagar la presencia de parafunciones diurnas como onicofagia, morderse los labios o las mejillas (36), morder objetos como lápices o bolígrafos (37) y el hábito de masticar chicles (38). Se debe considerar que de todas las parafunciones, se ha reportado que la onicofagia en vigilia es la más común (39).

En el examen clínico se considera evaluar la presencia de TTM como dolor en los músculos masticatorios en reposo o durante la función mandibular, sonidos en la articulación témporomandibular (ATM) en forma de chasquido o crepitación y dolor a la palpación en la ATM (40). Se debe evaluar también la presencia de cefaleas, molestias musculares en la región orofacial, atrición dentaria anormal, fatiga, dolor y bloqueo mandibular al despertar, indentaciones en la lengua y las mejillas $(9,15,21)$. Cuando existe apretamiento se observan facetas de desgaste rugosas y cuando existe frotamiento se observan facetas de desgaste lisas, disminución de las coronas clínicas, desgaste en chanfle de los incisivos, pérdida de los bordes festoneados e hipersensibilidad dentaria $(10,26)$. El método gold estándar para el diagnóstico del bruxismo del sueño es la utilización de la polisomnografía (PSG) que brinda grabaciones del sueño e incluyen registros y señales de electroencefalograma, electromiograma, electrocardiograma y grabaciones simultáneas de audio y video. Sin embargo, sus desventajas son 
el costo, el tiempo requerido para la evaluación y el cambio del entorno que podría influenciar en el comportamiento real $(15,21)$.

El diagnóstico ha sido clasificado en bruxismo posible, cuando se confirma mediante un autoinforme basado en el cuestionario $\mathrm{y} / 0$ la anamnesis; probable, cuando se basa en el autoinforme y en el examen clínico; y definitivo, cuando se confirma con el autoinforme, el examen clínico y la PSG (11).

\section{TRATAMIENTO}

Debido a que el bruxismo es una actividad parafuncional multifactorial, el tratamiento debe estar enfocado en la identificación de los factores etiológicos y no solo en los signos y síntomas. Se debe iniciar con información y educación a los padres y de allí continuar con técnicas de relajación en el niño y en el adolescente. Se recomiendan tres estrategias a nivel psico-conductual, dental y farmacológico (25).

\section{PSICO-CONDUCTUAL}

Se recomienda psicoterapia en base a asesoramiento, hipnosis, fitness, ejercicios de relajación y biofeedback (bioretroalimentación) (28). También se considera el uso de métodos eléctricos como estímulos electrolíticos para la relajación muscular. En ausencia de factores sistémicos se recomiendan ejercicios orales para desensibilización, uso de sensibilidad térmica, asesoramiento nutricional, suplemento de vitaminas y minerales (25). También se debe enseñar al niño y al adolescente a reconocer condiciones musculares de tensión y relajación (23).

\section{DENTAL}

Algunos autores reportan que las férulas oclusales en niños no han demostrado efectividad clara y podrían tener efectos nocivos en el crecimiento maxilar y en la erupción de los dientes permanentes (41). Su uso queda limitado para los casos más graves y cuando exista un adecuado control de los pacientes (42). La pérdida excesiva de tejido dental, hace necesaria la utilización de restauraciones temporales 0 definitivas usando técnicas directas, indirectas o coronas pediátricas (7).

\section{FARMACOLÓGICO}

Algunos estudios han reportado que el uso de benzodiazepinas (diazepam), relajantes musculares (metocarbomol), precursores de catecolaminas (L-dopa), antagonistas beta-adrenérgicos (propanolol) (43), clonidina, clonazepam (44) y toxinas butolínicas (45), reducen la frecuencia del BS en adultos. En la población pediátrica se reporta el uso de hidroxicina (46); sin embargo, la evidencia aún no es concluyente sobre su eficacia. Por este motivo, tales medicamentos deben ser utilizados con cautela prefiriendo otros manejos más conservadores.

\section{CONCLUSIONES}

En los niños de 3 hasta 506 años se maneja el concepto de desgaste dental fisiológico pero la dificultad es determinar cuál es el límite entre lo fisiológico y lo parafuncional. El desgaste dentario sólo, sin dolor o molestias, no implica necesariamente la presencia de bruxismo en los niños y adolescentes. Debido a que la etiología del bruxismo es multifactorial, el conocimiento de los factores de riesgo es útil para que el odontólogo brinde un diagnóstico efectivo basado en el cuestionario a los padres y en el examen clínico del niño. El tratamiento debe ser lo menos invasivo posible iniciándose con una estrategia psico-conductual, evitando el uso farmacológico que quedará reservado para los casos más complejos. Lo ideal es establecer una terapia multidisciplinaria entre el pediatra, odontólogo, psicólogo, fonoaudiólogo, padres y el propio paciente motivado. 


\section{REFERENCIAS}

1. Lavigne G. J., Rompré P. H., Montplaisir J. Y. Sleep bruxism: validity of clinical research diagnostic criteria in a controlled polysomnographic study. J Dent Res. 1996; 75 (1): 546-552.

2. American Academy of Sleep Medicine. The International Classification Of Sleep Disorders, Revised: Diagnostic and Coding Manual. 3rd ed. Westchester, American Academy of Sleep Medicine, 2014.

3. Guo H., Wang T., Li X., Ma Q., Niu X., Qui J. What sleep behaviors are associated with bruxism in children? A systematic review and meta-analysis. Sleep Breath. 2017; 21 (4): 1013-1023.

4. Lam M. H., Zhang J., Li A. M., Wing Y. K. A community study of sleep bruxism in Hong Kong children: association with comorbid sleep disorders and neurobehavioral consequences. Sleep Med. 2011; 12 (7): 641-645.

5. Firmani M., Reyes M., Becerra N., Flores G., Weitzman M., Espinosa P. Bruxismo de sueño en niños y adolescentes. Rev Chil Pediatr. 2015; 86 (5): 373-379.

6. Rojas D. A., Terán K. N., Álvarez C. Bruxismo del sueño en niños: actualización de la literatura. Odontol Pediatr. 2017; 25 (3): 209-216.

7. Fernández O. C. Bruxismo en niños. Salud Mil. 2016; 35 (2): 28-37.

8. Manfredini D., Lobbezoo F. Role of psychosocial factors in the etiology of bruxism. J Orofac Pain. 2009; 23 (2): 153-166.

9. De Luca Canto G., Singh V., Conti P., Dick B. D., Gozal D., Major P. W., FloresMir C. Association between sleep bruxism and psychosocial factors in children and adolescents: a systematic review. Clin Pediatr (Phila). 2015; 54 (5): 469-478.

10. Luiz M., Luiz T., Provenzano M., Takahashi K., Fracasso M. Bruxismo em Odontopediatria. Rev Dens. 2008; 16 (2): 14-20.

11. Lobbezoo F., Ahlberg J., Glaros A. G., Kato T., Koyano K., Lavigne G. J., de Leeuw R., Manfredini D., Svensson P., Winocur E. Bruxism defined and graded: an international consensus. J Oral Rehabil. 2013; 40 (1): 2-4.

12. Kato T., Lavigne G. J. Sleep bruxism: A sleep-related movement disorder. Sleep Med Clin. 2010; 5 (1): 9-35.

13. Carlsson G., Magnusson T. Bruxism and other oral parafunctions. En: Management of temporomandibular disorders in the general dental practice. Chicago: Quintessence, 1999. p. 33-42.

14. Manfredini D., Restrepo C., Diaz-Serrano K., Winocur E., Lobbezoo F. Prevalence of sleep bruxism in children: a systematic review of the literature. J Oral Rehabil. 2013; 40 (8): 631-642.

15. Machado E., Del Fabbro C., Cunali P. A., Kaizer O. B. Prevalence of sleep bruxism in children: a systematic review. Dental Press J Orthod. 2014; 19 (6): 54-61.

16. Shinkai R. S., Santos L. M., Silva F. A., Santos M. N. Prevalence of nocturnal bruxism 2-11-year-old children. Rev Odontol Univ São Paulo. 1998; 12 (1): 29-37.

17. Castelo P. M., Gavião M. B., Pereira L. J., Bonjardim L. R. Relationship between oral parafunctional/nutritive sucking habits and temporomandibular joint dysfunction in primary dentition. Int J Paediatr Dent. 2005; 15 (1): 29-36. 
18. Serra-Negra J. M., Paiva S. M., Seabra A. P., Dorella C., Lemos B. F., Pordeus I. A. Prevalence of sleep bruxism in a group of Brazilian schoolchildren. Eur Arch Paediatr Dent. 2010; 11 (4): 192-195.

19. Renner A. C., da Silva A. A., Rodriguez J. D., Simões V. M., Barbieri M. A., Bettiol H., Thomaz E. B., Saraiva Mda C. Are mental health problems and depression associated with bruxism in children? Community Dent Oral Epidemiol. 2012; 40 (3): 277-287.

20. Clementino M. A., Siqueira M. B., SerraNegra J. M., Paiva S. M., Granville-Garcia A. F. The prevalence of sleep bruxism and associated factors in children: a report by parents. Eur Arch Paediatr Dent. 2017; 18 (6): 399-404.

21. Sandoval H., Fariña M. P. Prevalencia de bruxismo del sueño en niños y su relación con los signos de trastornos temporomandibulares y las parafunciones diurnas. Int J Odontostomat. 2016; 10 (1): 41-47.

22. Lobbezoo F., Lavigne G. J. Do bruxism and temporomandibular disorders have a causeand-effect relationship? J Orofac Pain. 1997; 11 (1): 15-23.

23. Restrepo C. C., Alvarez E., Jaramillo C., Vélez C., Valencia I. Effects of psychological techniques on bruxism in children with primary teeth. J Oral Rehabil. 2001; 28 (4): 354-360.

24. Meklas J. F. Bruxism: diagnosis and treatment. J Acad Gen Dent. 1971; 19: 31-36.

25. Diniz M. B., Silva R. C., Zuanon A. C. Bruxismo na infância: um sinal de alerta para odontopediatras e pediatras. Rev Paul Pedistr. 2009; 27 (3): 329-334.

26. Antonio A. G., Pierro V. S., Maia L. C. Bruxism in children: a warning sign for psychological problems. J Can Dent Assoc. 2006; 72 (2): 155-160.

27. Ferreira M. I., Toledo O. A. Relação entre tempo de aleitamento materno e hábitos bucais. Rev ABO Nac. 1997; 5: 317-320.
28. Attanasio R. Nocturnal bruxism and its clinical management. Dent Clin North Am. 1991; 35 (1): 245-252.

29. Abe K., Shimakawa M. Genetic and developmental aspects of sleeptalking and teeth-grinding. Acta Paedopsychiatr. 1966; 33 (11): 339-344.

30. Glaros A. G. Incidence of diurnal and nocturnal bruxism. J Prosthet Dent. 1981; 45 (5): 545-549.

31. Junqueira T. H., Nahás-Scocate A. C., Valle-Corotti K. M., Conti A. C., Trevisan $\mathrm{S}$. Association of infantile bruxism and the terminal relationships of the primary second molars. Braz Oral Res. 2013; 27 (1): 42-47.

32. Castroflorio T., Bargellini A., Rossini G., Cugliari G., Rainoldi A., Deregibus A. Risk factors related to sleep bruxism in children: A systematic literature review. Arch Oral Biol. 2015; 60 (11): 1618-1624.

33. Kuhn M., Türp J. C. Risk factors for bruxism. Swiss Dent J. 2018;128 (2): 118-124.

34. Weideman C. L., Bush D. L., Yan-Go F. L., Clark G. T., Gornbein J. A. The incidence of parasomnias in child bruxers versus nonbruxers. Pediatr Dent. 1996; 18 (7): 456-460.

35. Marks M. B. Bruxism in allergic children. Am J Orthod. 1980; 77 (1): 48-59.

36. Carlsson G. E., Egermark I., Magnusson T. Predictors of bruxism, other oral parafunctions, and tooth wear over a 20-year follow-up period. J Orofac Pain. 2003; 17 (1): 50-57.

37. Vieira-Andrade R. G., Drumond C. L., Martins-Júnior P. A., Correa-Faria P., Gonzaga G. C., Marques L. S. Prevalence of sleep bruxism and associated factors in preschool children. Pediatr Dent. 2014; 36 (1): 46-50.

38. Cortese S. G., Fridman D. E., Farah C. L., Bielsa F., Grinberg J., Biondi A. M. Frequency of oral habits, dysfunctions, and personality traits in bruxing and nonbruxing children: a comparative study. Cranio. 2013; 31 (4): 283-290. 
39. Farsi N. M. Symptoms and signs of temporomandibular disorders and oral parafunctions among Saudi children. J Oral Rehabil. 2003; 30 (12): 1200-1208.

40. Restrepo C. C., Vásquez L. M., Alvarez M., Valencia I. Personality traits and temporomandibular disorders in a group of children with bruxing behavior. J Oral Rehabil. 2008; 35 (8): 585-593.

41. Restrepo C. C., Medina I., Patiño I. Effect of occlusal splints on the temporomandibular disorders, dental wear and anxiety of bruxist children. Eur J Dent. 2011; 5 (4): 441-450.

42. Carra M. C., Huynh N., Lavigne G. Sleep bruxism: a comprehensive overview for the dental clinician interested in sleep medicine. Dent Clin North Am. 2012; 56 (2): 387-413.
43. Macaluso G. M., Guerra P., Di Giovanni G., Boselli M., Parrino L., Terzano M. G. Sleep bruxism is a disorder related to periodic arousals during sleep. J Dent Res. 1998; 77: 565-573.

44. Huynh N., Manzini C., Rompre P. H., Lavigne G. J. Weighing the potential effectiveness of various treatments for sleep bruxism. J Can Dent Assoc. 2007; 73: 727-730.

45. Long H., Liao Z., Wang Y., Liao L., Lai W. Efficacy of botulinum toxins on bruxism: an evidence-based review. Int Dent J. 2012; 62 (1): $1-5$.

46. Ghanizadeh A., Zare S. A. preliminary randomised double-blind placebo-controlled clinical trial of hydroxyzine for treating sleep bruxism in children. J Oral Rehabil. 2013; 40 (6): 413-417. 